\title{
Effects of the Combination of Evogliptin and Leucine on Insulin Resistance and Hepatic Steatosis in High-Fat Diet-Fed Mice
}

\author{
Chang Yell Shin, Hak Yeong Lee, Gil Hyung Kim, Sun Young Park, Won Seok Choi and Uy Dong Sohn* \\ Department of Pharmacology, College of Pharmacy, Chung-Ang University, Seoul 06974, Republic of Korea
}

\begin{abstract}
In this study, we aimed to investigate the effects of 8 weeks of treatment with a combination of evogliptin and leucine, a branchedchain amino acid, in mice with high-fat diet (HFD)-induced diabetes. Treatment with evogliptin alone or in combination with leucine reduced the body weight of the mice, compared to the case for those from the HFD control group. Long-term treatment with evogliptin alone or in combination with leucine resulted in a significant reduction in glucose intolerance; however, leucine alone did not affect postprandial glucose control, compared to the case for the mice from the HFD control group. Furthermore, the combination of evogliptin and leucine prevented HFD-induced insulin resistance, which was associated with improved homeostasis model assessment for insulin resistance, accompanied by markedly reduced liver fat deposition, hepatic triglyceride content, and plasma alanine aminotransferase levels. The combination of evogliptin and leucine increased the gene expression levels of hepatic peroxisome proliferator-activated receptor alpha, whereas those of the sterol regulatory element-binding protein 1 and stearoyl-CoA desaturase 1 were not altered, compared to the case in the HFD-fed mice $(p<0.05)$. Thus, our results suggest that the combination of evogliptin and leucine may be beneficial for treating patients with type 2 diabetes and hepatic steatosis; however, further studies are needed to delineate the molecular mechanisms underlying the action of this combination.
\end{abstract}

Key Words: Evogliptin, Leucine, High-fat diet, Insulin resistance

\section{INTRODUCTION}

Mice with high-fat diet (HFD)-induced diabetes are characterized by insulin resistance and hepatic steatosis (Kim et al., 2012; Kim et al., 2017a); insulin resistance can aggravate hepatic steatosis (Browning and Horton, 2004; Perry et al., 2014). Prolonged hyperglycemia and obesity can result in insulin resistance, thereby leading to enhanced lipogenesis and fatty acid uptake with reduced fatty acid oxidation and hepatic triglyceride secretion, which consequently leads to the worsening of hepatic steatosis (Koo, 2013; Aroor et al., 2015).

Dipeptidyl peptidase 4 (DPP4) is a serine protease that inactivates various endogenous peptide substrates. Increased hepatic DPP4 expression is pathophysiologically linked to insulin resistance and non-alcoholic fatty liver disease (NAFLD) progression (Balaban et al., 2007; Lamers et al., 2011; Miyazaki et al., 2012; Williams et al., 2015). DPP4 inhibitors block the degradation of biologically active glucagon-like peptide-1 (GLP-1). Thus, although DPP4 inhibitors might show therapeutic potential for the treatment of NAFLD (Armstrong et al., 2013), it remains unclear where there is a direct causal relationship between DPP4 inhibitors and NAFLD.

Branched-chain amino acids (BCAAs), particularly, leucine, have been suggested to play roles in obesity and metabolic syndrome (Layman and Walker, 2006). Leucine is an essential BCAA that cannot be produced by the human body and can only be obtained from the diet (Zhang et al., 2007). Previous studies have suggested that leucine stimulates GLP-1 secretion (Chen and Reimer, 2009) and reduces the increase in blood glucose levels when co-ingested with glucose (Kalogeropoulou et al., 2008).

Potential pharmacodynamic interactions between DPP4 inhibitors and BCAAs have not yet been evaluated; thus, the mechanisms underlying the combined effects of DPP4 inhibitors and leucine on the blood glucose levels remain unclear. Leucine has been shown to promote GLP-1 secretion from the intestine (Chen and Reimer, 2009), and evogliptin can prolong the action of GLP-1 via the inhibition of DPP4 activity (Kim et al., 2012). Therefore, the combination of evogliptin and leucine may improve glucose metabolism, thereby ameliorating

\section{Open Access https://doi.org/10.4062/biomolther.2021.003}

This is an Open Access article distributed under the terms of the Creative Commons Attribution Non-Commercial License (http://creativecommons.org/licenses/by-nc/4.0/) which permits unrestricted non-commercial use, distribution, and reproduction in any medium, provided the original work is properly cited.
Received Jan 6, 2021 Revised Feb 9, 2021 Accepted Feb 27, 2021

Published Online Apr 5, 2021

\section{*Corresponding Author}

E-mail: udsohn@cau.ac.kr

Tel: +82-2-820-5614, Fax: +82-2-826-8752 
insulin resistance and NAFLD.

Therefore, the aim of this study was to evaluate the pharmacodynamic interactions between evogliptin and leucine in mice with HFD-induced diabetes.

\section{MATERIALS AND METHODS}

\section{Materials}

Evogliptin ((R)-4-[(R)-3-amino-4-(2,4,5-trifluorophenyl)butanoyl]-3-(t-butoxymethyl) piperazin-2-one) l-tartrate salt was synthesized by Dong-A ST (Yongin, Korea), and I-leucine was purchased from Sigma-Aldrich (St. Louis, MO, USA). All other chemicals were purchased from Sigma-Aldrich, unless otherwise stated.

\section{Animals}

All animal experiments were performed in accordance with, and approved by, the Institutional Animal Care and Use Committee of the Chung-Ang University (Seoul, Korea; IACUC-201700056). Male ICR mice were obtained from RaonBio (Yongin, Korea).

\section{HFD-induced diabetes mouse model}

At 6 weeks of age, ICR mice were fed an HFD (20\% protein, $35.5 \%$ fat, and $36.3 \%$ carbohydrates, w/w; Research Diets, New Brunswick, NJ, USA) ad libitum for 10 weeks. They were maintained at $21^{\circ} \mathrm{C}$ with a $12: 12-\mathrm{h}$ light/dark cycle at the animal facilities of the College of Pharmacy at the Chung-Ang University.

The HFD-fed mice (16-weeks-old) were housed (two mice/ cage). They were evenly divided on the basis of their blood glucose levels and body weights. The mice were treated with evogliptin as a diet admixture $(0.1 \%, w / w)$, leucine in drinking water $(1 \%, w / w)$, or a combination of evogliptin $(0.1 \%)$ and leucine $(1 \%)$ for 8 weeks ( $n=8 /$ group). The target free-form doses were approximately 100 and $1,000 \mathrm{mg} / \mathrm{kg} /$ day for $0.1 \%$ $(\mathrm{w} / \mathrm{w})$ evogliptin and $1 \%(\mathrm{w} / \mathrm{v})$ leucine, respectively. The mean administered doses were $40.6 \pm 1.9$ and $771.7 \pm 54.5 \mathrm{mg} / \mathrm{kg} /$ day for $0.1 \%(\mathrm{w} / \mathrm{w})$ evogliptin and $1 \%(\mathrm{w} / \mathrm{v})$ leucine, respectively. The non-fasting blood glucose levels, body weight, diet, and water consumption were measured on a weekly basis.

After eight weeks of treatment, the mice were fasted overnight, and blood was collected from the orbital venous sinus into heparin-filled tubes. The plasma was separated by centrifugation at $5,000 \mathrm{~g}$ for $5 \mathrm{~min}$.

\section{Oral glucose tolerance test (OGTT)}

An OGTT was conducted after 4 weeks of treatment with evogliptin, leucine, or their combination. The mice were fasted overnight, and basal blood glucose levels ( $t=-30 \mathrm{~min}$ ) were measured in the tail vein blood using a glucometer (AccuChek Active, Roche, Ireland). The mice were then challenged orally with a glucose solution $(2 \mathrm{~g} / 10 \mathrm{~mL} / \mathrm{kg})$, and blood glucose levels in the tail vein blood were measured at the indicated time points for $2 \mathrm{~h}$ after the glucose challenge. The blood glucose excursions are represented as the area under the curve $\left(\mathrm{AUC}_{0-2 h}\right)$.

\section{Insulin tolerance test (ITT)}

An ITT was conducted after six weeks of treatment with evogliptin, leucine, or their combination. The mice were fasted for $6 \mathrm{~h}$, and $0.75 \mathrm{U} / \mathrm{kg}$ human insulin (Sigma-Aldrich) was intraperitoneally injected. Blood glucose levels were measured in tail vein blood samples $0,15,30,60,90$, and 120 min after insulin injection. Data were processed and are expressed as percentages of the basal fasting glucose level, and the area under the normalized blood glucose-time curve is presented as $\mathrm{AUC}_{0-2 \mathrm{~h}}$ (\% $\left.\mathrm{min}\right)$.

\section{Histological examination}

Formalin-fixed liver tissue samples were processed, and $5-\mu \mathrm{m}$-thick paraffin sections were stained with hematoxylin and eosin (H\&E). The slides were examined under an IX71 microscope and photographed using a Cool Snap color digital camera (Olympus, Tokyo, Japan). All sections were examined by the same person, who was blinded to the treatment. At least three different liver sections were examined from eight mice from each treatment group. The scoring of lipid droplet accumulation in liver sections was conducted in a blinded manner using a scoring scale from 1 to 4 (minimal, mild, moderate, and severe). The relative area of the hepatic lipid droplets was assessed using Image-Pro Plus software ver. 5.1 (Media Cybernetics, Silver Spring, MD, USA).

\section{Biochemical parameters}

Plasma glucose, triglyceride (TG), total cholesterol, aspartate aminotransferase (AST), and alanine aminotransferase (ALT) levels were measured by enzymatic methods using a Model 20i® Analyzer (Konelab, Waltham, MA, USA). Plasma insulin levels were measured using an insulin enzyme-linked immunosorbent assay (ELISA) kit (Shibayagi, Gunma, Japan). Plasma total GLP-1 (KT-876) levels were analyzed using an ELISA kit (Epitope Diagnostics Inc., San Diego, CA, USA). Plasma glucagon levels were determined using a mouse/rat glucagon ELISA kit (YK090, Eagle Biosciences, Amherst, NH, USA). Insulin-sensitizing effects after the treatments were assessed using the homeostasis model assessment for insulin resistance (HOMA-IR; [plasma insulin level $(\mu \mathrm{U} / \mathrm{mL}) \times$ plasma glucose level (mmol/L)]/22.5) (Matthews et al., 1985).

\section{Real-time quantitative polymerase chain reaction (qPCR)}

Total RNA $(2 \mu \mathrm{g})$ was extracted from the tissues using a total RNA Mini Kit (W72070-150, Wizbiosolutions, Seongnam, Korea). The RNA was then reverse-transcribed using the RT Master system (W2203, Wizbiosolutions). Then, qPCR was performed using the QGreen 2X SYBR Green qPCR Master Mix (QG-05, Cellsafe, Yongin, Korea), following the manufacturer's instructions. The data were analyzed using the QIAGEN Rotor-Gene $Q$ Series software. The primers used are listed in Table 1.

\section{Data analysis}

The data are expressed as the mean \pm standard error of the mean (SEM). Statistical analyses were performed using SigmaStat 2.0 (SPSS, Chicago, IL, USA). Two-group comparisons were performed using the Student's $t$-test. For multigroup comparisons, one-way analysis of variance (ANOVA), followed by the Student-Newman-Keuls multiple comparison test, was used. Statistical significance was set at $p<0.05$. 


\section{RESULTS}

\section{Effects of evogliptin, leucine, and their combination on body weight gain}

We investigated the effects of eight weeks of treatment with evogliptin, leucine, and their combination on body weight gain on HFD-fed mice. Evogliptin alone and in combination with leucine continuously reduced HFD-induced body weight gain by $38 \%$ and $45 \%$, respectively, compared with that in HFD-fed control mice (Fig. 1A). However, leucine alone did not induce body weight reduction ( $+6 \%$ vs. HFD-fed control mice). There were no significant differences in food and water consumption

Table 1. Primers used for quantitative real-time PCR

\begin{tabular}{ll}
\hline Target gene & \multicolumn{1}{c}{ Forward/reverse primers } \\
\hline 3-actin & Forward: 5'-CGCCACCAGTTCGCCATGGA-3' \\
& Reverse: 5'-TACAGCCCGGGGAGCATCGT-3' \\
SREBP-1C & Forward: 5'-CAAGGCCATCGACTACATCCG-3' \\
& Reverse: 5'-CACCACTTCGGGTTTCATGC-3' \\
SCD-1 & Forward: 5'-TTCTTGCGATACACTCTGGTGC-3' \\
& Reverse: 5'-CGGGATTGAATGTTCTTGTCGT-3' \\
PPAR-a & Forward: 5'-ATTCTTACCTGTGAACACGACCTG-3' \\
& Reverse: 5'-GGGTTGTTGCTGGTCTTTCC-3' \\
PEPCK & Forward: 5'-CTGCATAACGGTCTGGACTTC-3' \\
& Reverse: 5'-CAGCAACTGCCCGTACTCC-3' \\
G6Pase & Forward: 5'-AAAAAGCCAACGTATGGATTCCG-3' \\
& Reverse: 5'-CAGCAAGGTAGATCCGGGA-3' \\
\hline
\end{tabular}

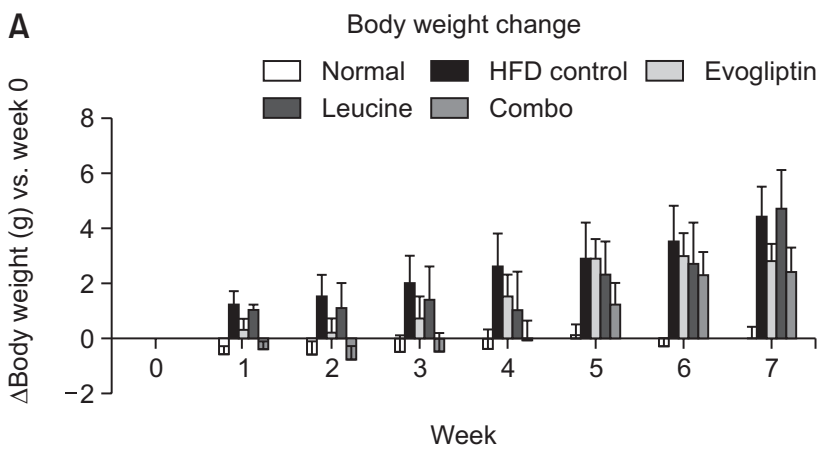

C

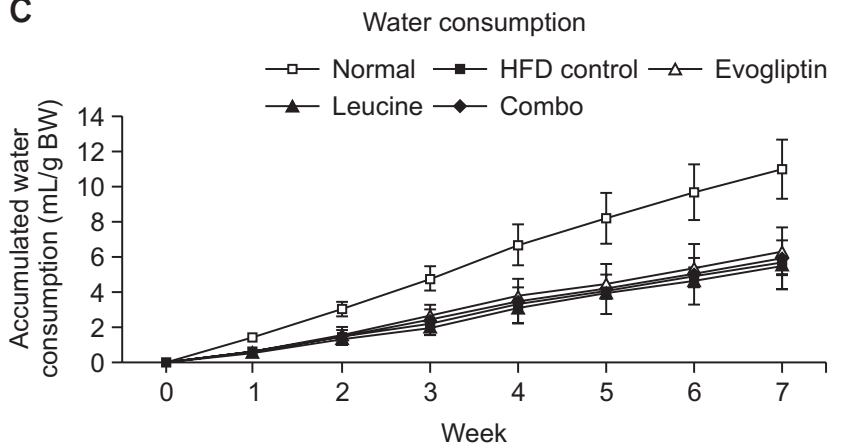

among the groups (Fig. 1B, 1C). Non-fasting blood glucose levels did not differ among the different treatment groups (Fig. 1D). These results showed that evogliptin or a combination of evogliptin and leucine prevented HFD-induced body weight gain without altering the food and water consumption.

\section{Effects of evogliptin, leucine, and their combination on postprandial glycemic control in HFD-fed mice}

To confirm the beneficial effects of the combination treatment on glycemic control in HFD-fed mice, we performed an OGTT four weeks after the drug treatments. The HFD-fed control mice exhibited a significantly higher glucose AUC than the normal control mice, which indicates severe glucose intolerance in the HFD-fed mice (Fig. 2A, 2B). Mice treated with evogliptin showed a $19.6 \%$ reduction in the glucose AUC; however, the leucine-treated mice did not show a significant change in glucose AUC (Fig. 2B). The addition of leucine augmented the glucose level-lowering effects of evogliptin $(-30.6 \%$ vs. HFD-fed control mice; $p<0.05)$. These results showed that long-term combination treatment with evogliptin and leucine improved glucose tolerance in mice with HFD-induced diabetes.

\section{Effects of evogliptin, leucine, and their combination on insulin resistance in HFD-fed mice}

To confirm the beneficial effects of the combination therapy on insulin resistance in HFD-fed mice, we performed an ITT six weeks after the drug treatments. The HFD-fed control mice exhibited a significantly higher glucose AUC than the normal control mice, following the normalization of the glucose levels to the basal blood glucose level (Fig. 3A). Mice treated
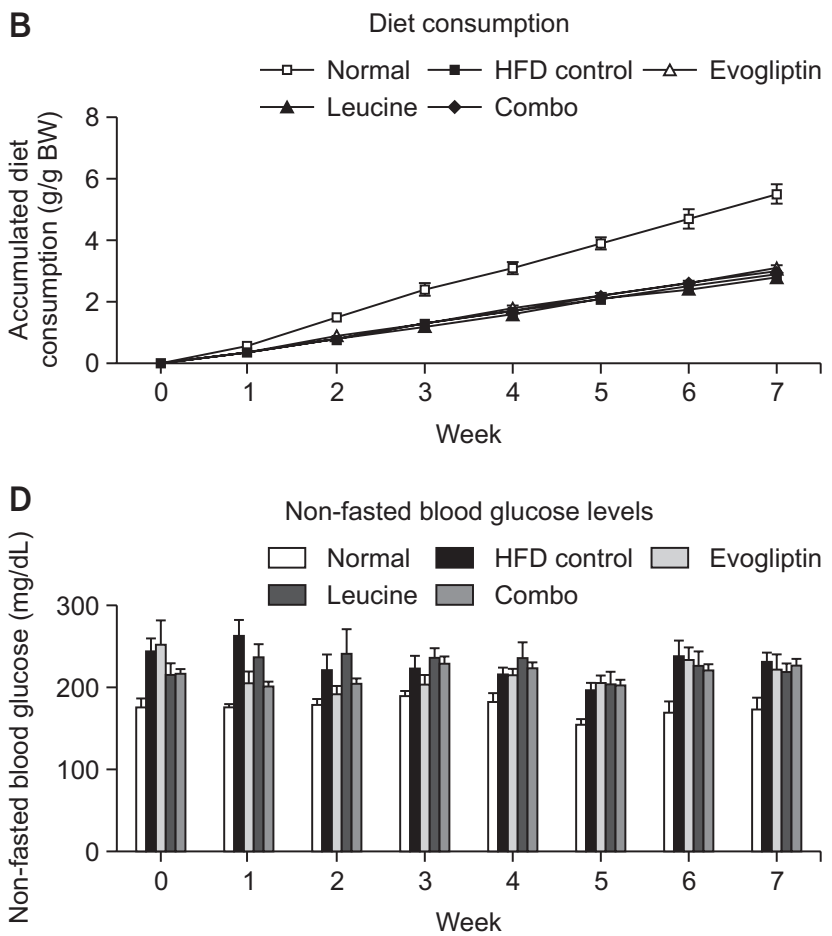

Fig. 1. Effects of eight weeks of treatment with evogliptin, leucine, and the combination of these two agents in HFD-fed mice. Body weight changes $(A)$, diet intake (B), water intake (C), and non-fasting blood glucose levels (D) were measured weekly. Data are expressed as the means \pm SEM. $p<0.05$ vs. the baseline (paired $t$-test). 
with $0.1 \%$ evogliptin or $1 \%$ leucine did not show significant improvements in insulin resistance despite $11.3 \%$ and $4.7 \%$ decreases in the glucose AUC, respectively, compared with the HFD-fed control mice. However, the combination of evogliptin and leucine resulted in a significant decrease in insulin resistance, with a $22.1 \%$ decrease in the glucose AUC, compared with that in the HFD-fed control mice (Fig. 3B).

After eight weeks of treatment, the plasma glucose levels in the mice treated with the combination of evogliptin and leucine did not significantly differ from those in the mice from the HFDfed control group (Fig. 3C). Plasma insulin levels tended to decrease in the evogliptin-treated mice, whereas leucine did not notably affect the plasma insulin levels (Fig. 3D). The combination treatment resulted in markedly decreased plasma insulin levels and improved HOMA-IR compared with those in
A

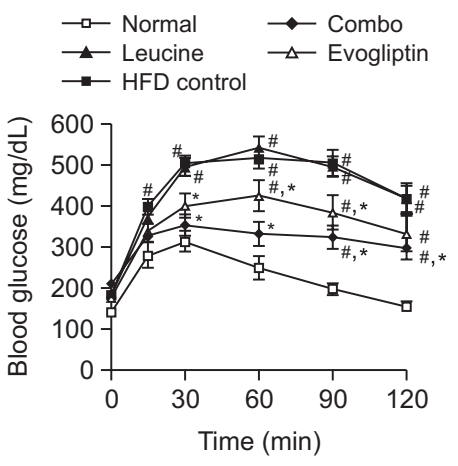

B

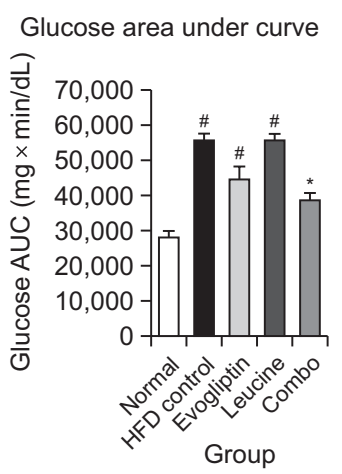

Fig. 2. Effects of evogliptin, leucine, and the combination of these two agents on postprandial glycemic control in HFD-fed mice. Blood glucose levels were measured $0,15,30,60,90$, and 120 min after glucose challenge $(A)$. Blood glucose $A_{U C} C_{0-2 h}$ was determined, and the percentage inhibition values were determined $(B)$. Data are expressed as the means \pm SEM. ${ }^{\#} p<0.05$ vs. normal control mice, ${ }^{*} p<0.05 v s$. HFD-fed control mice. the HFD-fed control mice (Fig. 3E). These results showed that long-term combination treatment with evogliptin and leucine prevented HFD-induced insulin resistance.

\section{Effects of evogliptin, leucine, and their combination on tissue weight and biochemical parameters}

We next examined the effects of eight weeks of treatment with evogliptin, leucine, and their combination on lipid dysregulation. The weights of the liver and epididymal fat were not significantly altered by evogliptin or leucine treatment (Table 2). The HFD-fed control mice showed notable changes in the plasma total cholesterol, glucagon, and ALT levels compared with the normal mice. The HFD-fed mice showed markedly elevated plasma ALT levels, which indicated hepatocyte damage owing to lipid accumulation. However, the HFD-fed control mice did not show a significant change in the plasma TG levels compared with the normal mice (Table 3). Evogliptin and leucine alone did not affect the plasma ALT levels, which was consistent with the insulin resistance not being affected by treatment with each of the agents alone. However, the combination treatment resulted in a significant decrease in the plasma ALT levels (Table 3). Collectively, these results suggest that the combination of evogliptin and leucine prevents HFD-induced disturbances in lipid metabolism.

Table 2. Effects of various treatments on liver weight in HFD-fed mice

\begin{tabular}{lcrc}
\hline \multicolumn{1}{c}{ Group } & BW $(\mathrm{g})$ & \multicolumn{1}{c}{ Liver $(\mathrm{g})$} & \multicolumn{1}{c}{ Liver/BW } \\
\hline Normal control & $38.3 \pm 0.3$ & $1.74 \pm 0.04$ & $0.0454 \pm 0.0013$ \\
HFD control & $57.5 \pm 1^{\#}$ & $2.56 \pm 0.15$ & $0.0445 \pm 0.0027$ \\
Evogliptin & $56.5 \pm 0.8^{\#}$ & $2.66 \pm 0.15$ & $0.0469 \pm 0.0021$ \\
Leucine & $57.5 \pm 1.1^{\#}$ & $2.8 \pm 0.21$ & $0.0484 \pm 0.0032$ \\
Combination & $54.6 \pm 1.5^{\#}$ & $2.31 \pm 0.13$ & $0.0422 \pm 0.0016$ \\
\hline
\end{tabular}

Data are expressed as the means \pm SEM. ${ }^{\#} p<0.05$ vs. normal control mice.

Insulin tolerance test
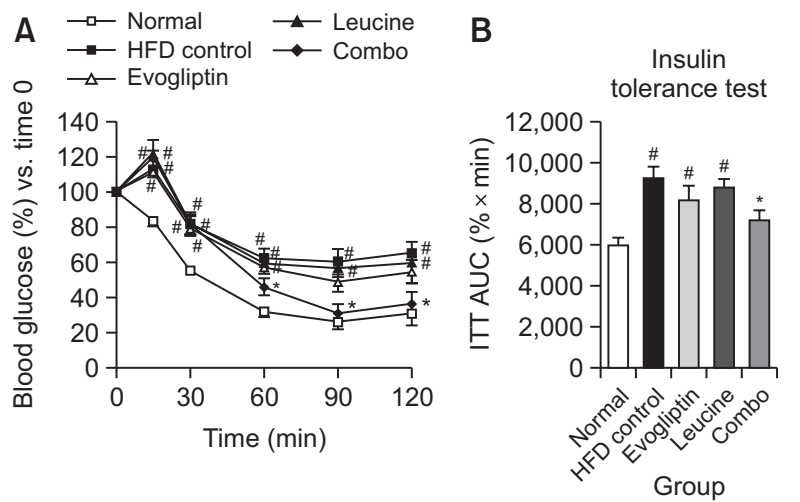

C

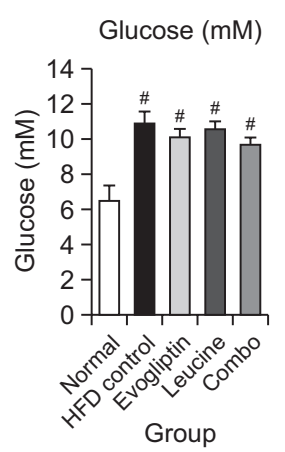

D

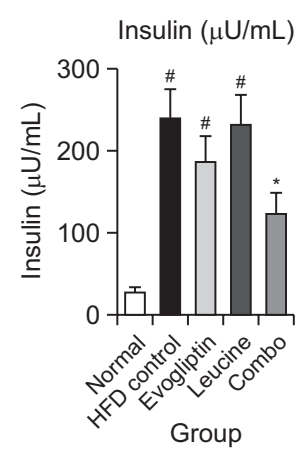

E

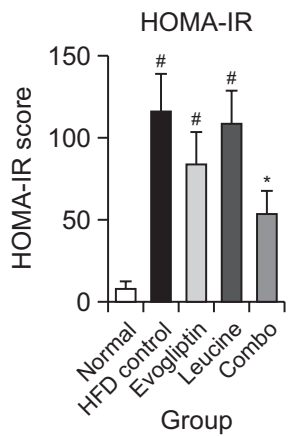

Fig. 3. Effects of evogliptin, leucine, and the combination of these two agents on insulin resistance and postprandial glycemic control in HFD-fed mice. Blood glucose changes were determined for $2 \mathrm{~h}(\mathrm{~A})$, and the blood glucose excursion over $2 \mathrm{~h}$ is presented (B). At week 8 , the animals were euthanized, and both fasting plasma glucose (C) and plasma insulin (D) levels were measured. Homeostasis model assessment for insulin resistance (HOMA-IR) was improved by the combination treatment (E). Data are expressed as the means \pm SEM. ${ }^{\#} p<0.05$ vs. normal control mice, ${ }^{*} p<0.05$ vs. HFD-fed control mice. 
Table 3. Effects of various treatments on biochemical parameters in HFD-fed mice

\begin{tabular}{lllcrrr}
\hline Group & ALT $(\mathrm{U} / \mathrm{L})$ & AST $(\mathrm{U} / \mathrm{L})$ & $\begin{array}{c}\text { Triglycerides } \\
(\mathrm{mg} / \mathrm{dL})\end{array}$ & $\begin{array}{c}\text { Total cholesterol } \\
(\mathrm{mg} / \mathrm{dL})\end{array}$ & Total GLP-1 (pM) Glucagon $(\mathrm{pg} / \mathrm{mL})$ \\
\hline Lean & $43.7 \pm 4.8$ & $122.3 \pm 5.8$ & $154.1 \pm 27.6$ & $129 \pm 5.5$ & $2.74 \pm 0$ & $414.3 \pm 14.5$ \\
HFD control & $80.2 \pm 11.8^{\#}$ & $127.3 \pm 8$ & $130.5 \pm 9.8$ & $246.2 \pm 19^{\#}$ & $2.71 \pm 0.01$ & $901.4 \pm 82^{\#}$ \\
Evogliptin & $67.5 \pm 7.3^{\#}$ & $126.4 \pm 4.7$ & $114.1 \pm 7.7$ & $291.6 \pm 11.2^{\#}$ & $2.77 \pm 0.01$ & $753.7 \pm 93.3^{\#}$ \\
Leucine & $80.9 \pm 8.3^{\#}$ & $156.3 \pm 9$ & $114.8 \pm 5.2$ & $283.8 \pm 12.5^{\#}$ & $2.9 \pm 0.02$ & $870.2 \pm 115.6^{\#}$ \\
Combination & $46.5 \pm 6.2^{*}$ & $134.6 \pm 7.3$ & $136.1 \pm 12.5$ & $287 \pm 15.7^{\#}$ & $3.25 \pm 0.34$ & $882 \pm 74^{\#}$ \\
\hline
\end{tabular}

Data are expressed as the means \pm SEM. ${ }^{*} p<0.05$ vs. normal control mice, ${ }^{*} p<0.05$ vs. HFD-fed control mice.
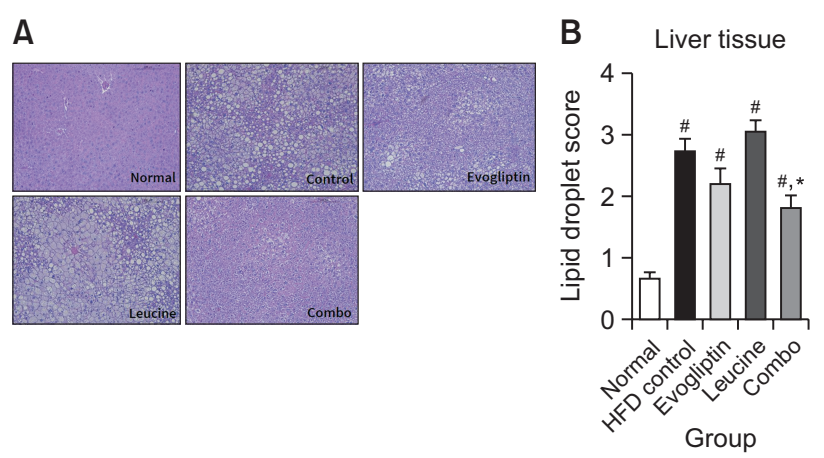

Fig. 4. Effects of evogliptin, leucine, and the combination of these two agents on hepatic steatosis in HFD-fed mice. After eight weeks of the administration of evogliptin, leucine, or their combination in HFD-fed mice, the degree of hepatic steatosis was assessed by examining hepatic lipid accumulation histologically $(A)$ and biochemically (B). Data are expressed as the means \pm SEM. ${ }^{*} p<0.05$ vs. normal control mice, ${ }^{*} p<0.05$ vs. HFD-fed control mice.

\section{Histological examination of fat content in liver tissues from HFD-fed mice}

A histological examination was performed to measure the fat contents in H\&E-stained liver sections. The HFD-fed mice exhibited prominent adipocyte hypertrophy compared with the normal mice (Fig. 4). Evogliptin and leucine alone did not reduce the fat content; however, their combination markedly improved hepatic histology, as shown by quantitative histomorphometry analysis (Fig. 4).

\section{Chronic effects of evogliptin, leucine, and their combination on hepatic gene expression and intracellular signaling in HFD-fed mice}

Considering the importance of the liver in the regulation of systemic insulin sensitivity, we explored the effects of evogliptin, leucine, and their combination on the expression of genes involved in glucose and lipid metabolism. Sterol regulatory element-binding protein-1c (SREBP-1c) is a key transcription factor that regulates de novo lipogenesis under the control of insulin signaling (Yecies et al., 2011). To determine whether the reduction of hepatic TG accumulation by evogliptin and leucine treatment was mediated by the suppression of lipogenesis, we assessed the gene expression levels of SREBP-1c and stearoyl-CoA desaturase-1 (SCD-1), a downstream gene. Evogliptin, leucine, and their combination did not affect the expression of SREBP-1c, compared with that in the control mice (Fig. 5A). Similarly, the expression of SCD-1 was not significantly affected by the drug treatment (Fig. 5B). In the liver, peroxisome prolif- erator-activated receptor alpha (PPAR $\alpha$ ) is highly expressed, and its activation inhibits lipogenesis via both direct and indirect mechanisms. Next, we examined whether evogliptin, leucine, and their combination induce the upregulation of PPAR $\alpha$. Hepatic PPAR- $\alpha$ expression decreased by $60.4 \%$ in HFD-fed mice compared with the normal control mice. However, treatments with evogliptin and leucine alone normalized the PPAR $\alpha$ expression. Furthermore, the combination treatment significantly increased the expression of PPAR $\alpha$, compared with that in the HFD-fed control mice (Fig. 5C). These data suggest that the restoration or activation of PPAR $\alpha$ expression by the combination treatment partly contributes to the preventive effects of evogliptin and leucine against lipogenesis. We then examined whether evogliptin, leucine, and their combination suppress the expression of gluconeogenic genes. In the HFD-fed mice, the hepatic expression levels of glucose 6-phosphatase (G6Pase) and phosphoenolpyruvate carboxykinase (PEPCK), which are key genes involved in the regulation of hepatic gluconeogenesis, were not different from those in the normal control mice. Additionally, the drug treatments did not notably affect the mRNA expression of G6Pase and PEPCK; however, evogliptin alone and the combination treatment decreased the mRNA expression of G6Pase by $33.9 \%$ and 39\%, respectively (Fig. 5D, 5E).

\section{DISCUSSION}

In this study, the combination of evogliptin and leucine exhibited synergistic therapeutic effects against hepatic steatosis by decreasing insulin resistance and suppressing lipogenesis.

Although most DPP4 inhibitors have been reported to have neutral effects on body weight gain in animals and humans (Kern et al., 2012; Holst and Deacon, 2013; Omar et al., 2013), several DPP4 inhibitors have been shown to exhibit anti-obesity effects in mice with HFD-induced obesity (FukudaTsuru et al., 2014; Chae et al., 2015). In this study, there were no marked changes in body weight in mice treated with evogliptin or leucine alone, although their combination reduced the HFD-induced body weight gain. In a previous study (Kim et al., 2017a), there was a significant difference in body weight between HFD-fed and evogliptin-treated (0.5\%; $300 \mathrm{mg} / \mathrm{kg}$ ) mice, but not between HFD-fed and evogliptin-treated $(0.2 \%$; $100 \mathrm{mg} / \mathrm{kg}$ ) mice. Therefore, it seems that evogliptin (0.1\%) affected the DPP4 activity, but not to an extent that was sufficient enough to reduce body weight, which is consistent with the findings of a previous report (Chae et al., 2015). Zhang et al. (2007) reported that $1.5 \%$ leucine supplementation induces a $32 \%$ reduction in HFD-induced weight gain; however, in this study, $1 \%$ leucine supplementation did not affect body 

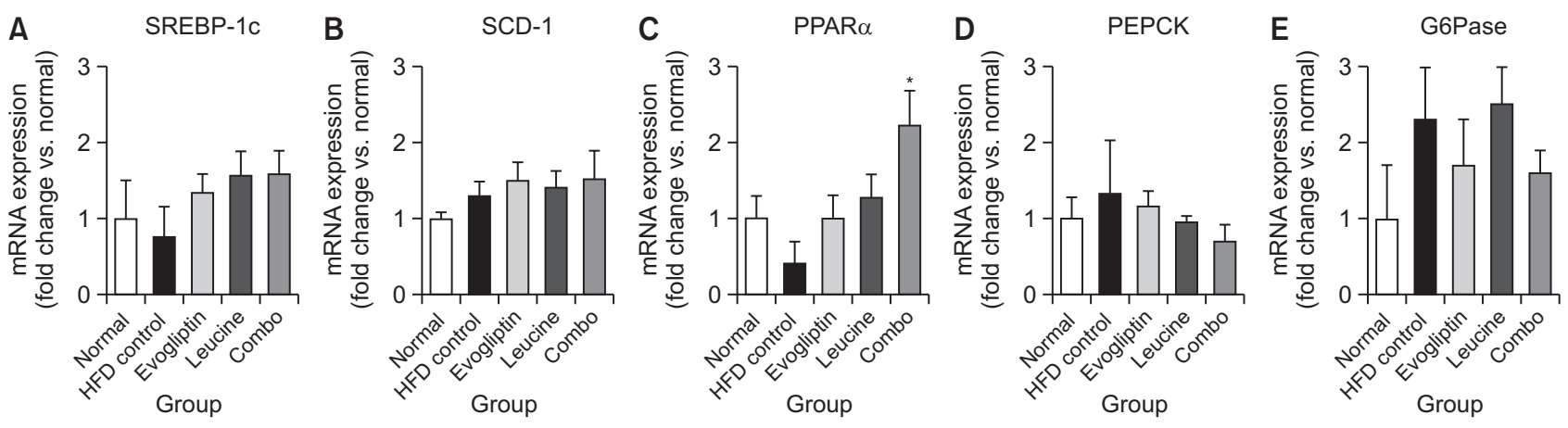

Fig. 5. Effects of evogliptin, leucine, and the combination of these two agents on gene expression in liver tissues. After eight weeks of the administration of evogliptin, leucine, or their combination, the gene expression levels of SREBP-1C (A), SCD-1 (B), PPAR $\alpha$ (C), PEPCK (D), and G6Pase (E) in the liver tissues of HFD-fed mice were assessed by RT-qPCR ( $n=8 /$ group). Data are expressed as the means \pm SEM. ${ }^{*} p<0.05$ vs. HFD-fed control mice.

weight. Thus, we concluded that $1 \%$ leucine does not notably affect food consumption and body weight, although it increased GLP-1 secretion (Chen and Reimer, 2009). However, the combination of evogliptin and leucine decreased the HFDinduced weight gain by $45 \%$, which indicates the synergistic effects of this combination on HFD-induced body weight gain.

Herein, the HFD-fed mice showed mild hyperglycemia and glucose and insulin intolerance, which is consistent with the results of a previous report (Kim et al., 2017a). By reducing the blood glucose levels, evogliptin improved glucose tolerance in insulin-resistant mice. HFD-induced postprandial hyperglycemia and insulin resistance were not markedly affected by the addition of leucine, which is consistent with the results of a previous report (Fu et al., 2015). However, the combination of leucine and evogliptin significantly decreased the postprandial glucose levels and the degree of insulin resistance in HFD-fed mice. Therefore, the synergistic effects of leucine and evogliptin on postprandial glycemic control and insulin sensitivity might be attributed to the evogliptin-induced inhibition of DPP4-mediated GLP-1 degradation (Kim et al., 2012) and the leucine-induced promotion of GLP-1 secretion from the intestine (Chen and Reimer, 2009).

Hepatic steatosis, a hallmark of NAFLD, results from the accumulation of lipids, particularly triglycerides, in the liver (Qin and Tian, 2010). The pathogenesis of NAFLD has not yet been clearly defined; however, adipose tissue dysfunction characterized by insulin resistance is considered a central mechanism involved in the development of steatosis and transition to steatohepatitis in NAFLD (Wang et al., 2010). The liver, which is the major organ responsible for storing excess carbohydrates by converting them to glycogen or lipids, disposes off $25-35 \%$ of the oral glucose load (Moore et al., 2012). Recent studies have shown that BCAAs play an important role in the regulation of energy balance and metabolism (Zhang et al., 2007; Macotela et al., 2011). For example, Chen et al. (2018) reported that leucine supplementation reduces insulin resistance and hepatic steatosis in $\mathrm{db} / \mathrm{db}$ mice, which suggests that dietary leucine supplementation is a potential nutritional intervention to attenuate hepatic steatosis and early diabetic neuropathy in type 2 diabetes. Although limited clinical data from non-randomized trials using small groups of patients with diabetes are available, several clinical studies have suggested that DPP4 inhibitors have beneficial effects on liver enzyme levels and histology in patients with NAFLD and type 2 diabetes (Iwasaki et al., 2011; Yilmaz et al., 2012; Mashitani et al., 2016). As insulin resistance contributes to the development of hepatic steatosis, the use of an agent that decreases insulin resistance could be an ideal treatment for hepatic steatosis. Accordingly, we aimed to evaluate the combined effects of DPP4 inhibitors and leucine on the development of hepatic steatosis. The combination of evogliptin and leucine significantly suppressed the progression of fatty liver. The marked reduction in the hepatic TG content was associated with a decrease in the plasma ALT level, a marker of hepatic infiltration that is frequently elevated in hepatic steatosis. These data suggest that a decrease in insulin resistance results in a reduction of the hepatic lipid content and plasma ALT levels. Because caloric intake plays an important role in the pathogenesis of insulin resistance and hepatic steatosis, we measured the mean daily food intake. Our results showed that daily food intake did not differ markedly among the groups, which eliminated the possible effects of hyperphagia on insulin resistance.

Lipogenesis is a key process that underlies hepatic lipid accumulation in HFD-fed mice (Kim et al., 2017a). DPP4 deficiency inhibits lipogenesis, thereby preventing hepatic steatosis in HFD-fed mice (Conarello et al., 2003). Leucine supplementation has also been reported to reduce hepatic steatosis in $\mathrm{db} / \mathrm{db}$ mice (Chen et al., 2018). PPAR $\alpha$ is highly expressed in the liver, and its activation stimulates the expression of genes related to peroxisomal $\beta$-oxidation (Rakhshandehroo et al., 2009). Additionally, PPAR $\alpha$ inhibits lipogenesis in the liver via both direct and indirect mechanisms (Kim et al., $2017 b)$. As the combination of evogliptin and leucine significantly prevented the formation of hepatic lipid droplets in the liver, we examined the expression of genes involved in hepatic lipogenesis and fatty acid oxidation to elucidate the mechanisms underlying the effects of this combination on hepatic steatosis. In this study, evogliptin did not affect the expression levels of SREBP-1C and SCD-1, which is consistent with the results of a previous study (Kim et al., 2017a). However, evogliptin and leucine alone restored PPAR $\alpha$ expression, although these effects were not statistically significant. Furthermore, the combination treatment elicited a significant increase in PPAR $\alpha$ expression, compared with that in HFD-fed control mice. Therefore, we suggest that the combination treatment 
reduces the degree of hepatic steatosis directly via the activation of PPAR $\alpha$ expression or indirectly via the reduction of lipid accumulation. However, further studies are required to elucidate the mechanisms underlying these processes.

In summary, our results showed that the combination of evogliptin and leucine ameliorated hepatic steatosis by improving insulin resistance and upregulating PPAR $\alpha$ expression. Therefore, this therapy might be beneficial in patients with type 2 diabetes and hepatic steatosis; however, more rigorous testing is needed to delineate the molecular mechanisms underlying the action of this combination.

\section{CONFLICT OF INTEREST}

The authors declare that there are no conflicts of interest.

\section{ACKNOWLEDGMENTS}

This research was supported by the Basic Science Research Program through the National Research Foundation of Korea (NRF), funded by the Ministry of Education, Science and Technology [Grant NRF-2019R1F1A1062070].

\section{REFERENCES}

Armstrong, M. J., Houlihan, D. D., Rowe, I. A., Clausen, W. H., Elbrond, B., Gough, S. C., Tomlinson, J. W. and Newsome, P. N. (2013) Safety and efficacy of liraglutide in patients with type 2 diabetes and elevated liver enzymes: individual patient data meta-analysis of the LEAD program. Aliment. Pharmacol. Ther. 37, 234-242.

Aroor, A. R., Habibi, J., Ford, D. A., Nistala, R., Lastra, G., Manrique, C., Dunham, M. M., Ford, K. D., Thyfault, J. P., Parks, E. J., Sowers, J. R. and Rector, R. S. (2015) Dipeptidyl peptidase-4 inhibition ameliorates Western diet-induced hepatic steatosis and insulin resistance through hepatic lipid remodeling and modulation of hepatic mitochondrial function. Diabetes 64, 1988-2001.

Balaban, Y. H., Korkusuz, P., Simsek, H., Gokcan, H., Gedikoglu, G., Pinar, A., Hascelik, G., Asan, E., Hamaloglu, E. and Tatar, G. (2007) Dipeptidyl peptidase IV (DDP IV) in NASH patients. Ann. Hepatol. 6, 242-250.

Browning, J. D. and Horton, J. D. (2004) Molecular mediators of hepatic steatosis and liver injury. J. Clin. Invest. 114, 147-152.

Chae, Y. N., Kim, T. H., Kim, M. K., Shin, C. Y., Jung, I. H., Sohn, Y. S. and Son, M. H. (2015) Beneficial effects of evogliptin, a novel dipeptidyl peptidase 4 inhibitor, on adiposity with increased Ppargc1a in white adipose tissue in obese mice. PLOS ONE 10, e0144064.

Chen, K. H., Chen, Y. L., Tang, H. Y., Hung, C. C., Yen, T. H., Cheng, M. L., Shiao, M. S. and Chen, J. K. (2018) Dietary leucine supplement ameliorates hepatic steatosis and diabetic nephropathy in $\mathrm{db} /$ db mice. Int. J. Mol. Sci. 19, 1921.

Chen, Q. and Reimer, R. A. (2009) Dairy protein and leucine alter GLP-1 release and mRNA of genes involved in intestinal lipid metabolism in vitro. Nutrition 25, 340-349.

Conarello, S. L., Li, Z., Ronan, J., Roy, R. S., Zhu, L., Jiang, G., Liu, F., Woods, J., Zycband, E., Moller, D. E., Thornberry, N. A. and Zhang, B. B. (2003) Mice lacking dipeptidyl peptidase IV are protected against obesity and insulin resistance. Proc. Natl. Acad. Sci. U.S.A. 100, 6825-6830.

Fu, L., Bruckbauer, A., Li, F., Cao, Q., Cui, X., Wu, R., Shi, H., Zemel, M. B. and Xue, B. (2015) Leucine amplifies the effects of metformin on insulin sensitivity and glycemic control in diet-induced obese mice. Metabolism 64, 845-856.

Fukuda-Tsuru, S., Kakimoto, T., Utsumi, H., Kiuchi, S. and Ishii, S. (2014) The novel dipeptidyl peptidase-4 inhibitor teneligliptin pre- vents high-fat diet-induced obesity accompanied with increased energy expenditure in mice. Eur. J. Pharmacol. 723, 207-215.

Holst, J. J. and Deacon, C. F. (2013) Is there a place for incretin therapies in obesity and prediabetes? Trends Endocrinol. Metab. 24, 145-152.

Iwasaki, T., Yoneda, M., Inamori, M., Shirakawa, J., Higurashi, T., Maeda, S., Terauchi, Y. and Nakajima, A. (2011) Sitagliptin as a novel treatment agent for non-alcoholic Fatty liver disease patients with type 2 diabetes mellitus. Hepatogastroenterology 58, 2103-2105.

Kalogeropoulou, D., Lafave, L., Schweim, K., Gannon, M. C. and Nuttall, F. Q. (2008) Leucine, when ingested with glucose, synergistically stimulates insulin secretion and lowers blood glucose. Metabolism 57, 1747-1752.

Kern, M., Kloting, N., Niessen, H. G., Thomas, L., Stiller, D., Mark, M., Klein, T. and Bluher, M. (2012) Linagliptin improves insulin sensitivity and hepatic steatosis in diet-induced obesity. PLOS ONE 7, e38744.

Kim, M. K., Chae, Y. N., Ahn, G. J., Shin, C. Y., Choi, S. H., Yang, E. K., Sohn, Y. S. and Son, M. H. (2017a) Prevention and treatment effect of evogliptin on hepatic steatosis in high-fat-fed animal models. Arch. Pharm. Res. 40, 268-281.

Kim, M. K., Chae, Y. N., Kim, H. D., Yang, E. K., Cho, E. J., Choi, S. H., Cheong, Y. H., Kim, H. S., Kim, H. J., Jo, Y. W., Son, M. H., Kim, S. H. and Shin, C. Y. (2012) DA-1229, a novel and potent DPP4 inhibitor, improves insulin resistance and delays the onset of diabetes. Life Sci. 90, 21-29.

Kim, S. M., Lee, B., An, H. J., Kim, D. H., Park, K. C., Noh, S. G., Chung, K. W., Lee, E. K., Kim, K. M., Kim, S. J., Chun, P., Lee, H. J., Moon, H. R. and Chung, H. Y. (2017b) Novel PPARalpha agonist MHY553 alleviates hepatic steatosis by increasing fatty acid oxidation and decreasing inflammation during aging. Oncotarget 8 , 46273-46285.

Koo, S. H. (2013) Nonalcoholic fatty liver disease: molecular mechanisms for the hepatic steatosis. Clin. Mol. Hepatol. 19, 210-215.

Lamers, D., Famulla, S., Wronkowitz, N., Hartwig, S., Lehr, S., Ouwens, D. M., Eckardt, K., Kaufman, J. M., Ryden, M., Muller, S., Hanisch, F. G., Ruige, J., Arner, P., Sell, H. and Eckel, J. (2011) Dipeptidyl peptidase 4 is a novel adipokine potentially linking obesity to the metabolic syndrome. Diabetes 60, 1917-1925.

Layman, D. K. and Walker, D. A. (2006) Potential importance of leucine in treatment of obesity and the metabolic syndrome. J. Nutr. 136, 319S-323S.

Macotela, Y., Emanuelli, B., Bang, A. M., Espinoza, D. O., Boucher, J., Beebe, K., Gall, W. and Kahn, C. R. (2011) Dietary leucine--an environmental modifier of insulin resistance acting on multiple levels of metabolism. PLoS ONE 6, e21187.

Mashitani, T., Noguchi, R., Okura, Y., Namisaki, T., Mitoro, A., Ishii, H., Nakatani, T., Kikuchi, E., Moriyasu, H., Matsumoto, M., Sato, S., An, T., Morita, H., Aizawa, S., Tokuoka, Y., Ishikawa, M., Matsumura, Y., Ohira, H., Kogure, A., Noguchi, K. and Yoshiji, H. (2016) Efficacy of alogliptin in preventing non-alcoholic fatty liver disease progression in patients with type 2 diabetes. Biomed. Rep. 4, 183187.

Matthews, D. R., Hosker, J. P., Rudenski, A. S., Naylor, B. A., Treacher, D. F. and Turner, R. C. (1985) Homeostasis model assessment: insulin resistance and beta-cell function from fasting plasma glucose and insulin concentrations in man. Diabetologia 28, 412-419.

Miyazaki, M., Kato, M., Tanaka, K., Tanaka, M., Kohjima, M., Nakamura, K., Enjoji, M., Nakamuta, M., Kotoh, K. and Takayanagi, R. (2012) Increased hepatic expression of dipeptidyl peptidase-4 in non-alcoholic fatty liver disease and its association with insulin resistance and glucose metabolism. Mol. Med. Rep. 5, 729-733.

Moore, M. C., Coate, K. C., Winnick, J. J., An, Z. and Cherrington, A. D. (2012) Regulation of hepatic glucose uptake and storage in vivo. Adv. Nutr. 3, 286-294.

Omar, B. A., Vikman, J., Winzell, M. S., Voss, U., Ekblad, E., Foley, J. E. and Ahren, B. (2013) Enhanced beta cell function and antiinflammatory effect after chronic treatment with the dipeptidyl peptidase-4 inhibitor vildagliptin in an advanced-aged diet-induced obesity mouse model. Diabetologia 56, 1752-1760.

Perry, R. J., Samuel, V. T., Petersen, K. F. and Shulman, G. I. (2014) The role of hepatic lipids in hepatic insulin resistance and type 2 
diabetes. Nature 510, 84-91

Qin, Y. and Tian, Y. P. (2010) Preventive effects of chronic exogenous growth hormone levels on diet-induced hepatic steatosis in rats. Lipids Health Dis. 9, 78.

Rakhshandehroo, M., Hooiveld, G., Muller, M. and Kersten, S. (2009) Comparative analysis of gene regulation by the transcription factor PPARalpha between mouse and human. PLoS ONE 4, e6796.

Wang, Z., Yao, T., Pini, M., Zhou, Z., Fantuzzi, G. and Song, Z. (2010) Betaine improved adipose tissue function in mice fed a high-fat diet: a mechanism for hepatoprotective effect of betaine in nonalcoholic fatty liver disease. Am. J. Physiol. Gastrointest. Liver Physiol. 298, G634-G642.

Williams, K. H., Vieira De Ribeiro, A. J., Prakoso, E., Veillard, A. S. Shackel, N. A., Brooks, B., Bu, Y., Cavanagh, E., Raleigh, J., McLennan, S. V., McCaughan, G. W., Keane, F. M., Zekry, A. Gorrell, M. D. and Twigg, S. M. (2015) Circulating dipeptidyl pep- tidase-4 activity correlates with measures of hepatocyte apoptosis and fibrosis in non-alcoholic fatty liver disease in type 2 diabetes mellitus and obesity: a dual cohort cross-sectional study. J. Diabetes 7, 809-819.

Yecies, J. L., Zhang, H. H., Menon, S., Liu, S., Yecies, D., Lipovsky, A. I., Gorgun, C., Kwiatkowski, D. J., Hotamisligil, G. S., Lee, C. H. and Manning, B. D. (2011) Akt stimulates hepatic SREBP1c and lipogenesis through parallel mTORC1-dependent and independent pathways. Cell Metab. 14, 21-32

Yilmaz, Y., Yonal, O., Deyneli, O., Celikel, C. A., Kalayci, C. and Duman, D. G. (2012) Effects of sitagliptin in diabetic patients with nonalcoholic steatohepatitis. Acta Gastroenterol. Belg. 75, 240-244.

Zhang, Y., Guo, K., LeBlanc, R. E., Loh, D., Schwartz, G. J. and Yu, Y. H. (2007) Increasing dietary leucine intake reduces diet-induced obesity and improves glucose and cholesterol metabolism in mice via multimechanisms. Diabetes 56, 1647-1654. 